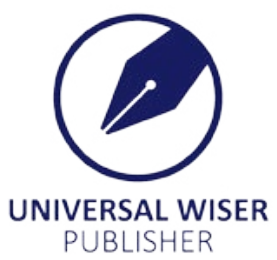

\title{
On-Package Color Indicator for Ripeness Monitoring of tape ketan Packaging
}

\author{
Kuswandi, B.*, Mahawan, A. G., Nuriman \\ Chemo and Biosensors Group, Faculty of Pharmacy, University of Jember, Jl. Kalimantan 37, Jember, 68121, Indonesia \\ *E-mail: b_kuswandi.farmasi@unej.ac.id
}

\begin{abstract}
A color indicator for tape ketan (TK) (Indonesian fermented steamed glutinous rice product) packaging was developed to monitor the fermentation stage of TK during storage and distribution. Tape ketan was packed in a tray of polypropylene (PP) with a lid of nylon/cast polypropylene (Ny/CPP). A color indicator film consisting of Nafion ${ }^{\circledR}$ and crystal violet $(\mathrm{CV})$ was attached to the lid as an on-package indicator and change its color as water vapor or relative humidity changes. During fermentation at ambient temperature $\left(28 \pm 1^{\circ} \mathrm{C}\right)$, the TK quality was assessed for $\mathrm{pH}$, titratable acidity (TA), ethanol content and sensory evaluation (aroma, taste, and color), in correlation with the color change of the indicator. In conclusion, the color values of the indicator correlated well with sensory values as a degree for fermentation stage of TK along with other parameters, such as $\mathrm{pH}$, TA, and ethanol, and could be detected clearly with yellow for unripe, blue for ripe, and finally pale blue for overripe.
\end{abstract}

Keywords: colorimetric method, smart packaging, sensor, ripeness, fermented food

\section{Introduction}

Tape ketan (TK) is a popular fermented steamed glutinous rice product in Indonesian, with a sweet taste with bit acid and flavor of the mild alcoholic. Usually, in Indonesia, TK is produced by the home industry and manufactured traditionally or home-made for the consumption of the family. TK is prepared from glutinous rice (Oryza sativa glutinosa) that has been steamed, then spread in thin layers on the trays made of woven bamboo, and then inoculated with ragi (dry flattened circular cakes) about $3 \mathrm{~cm}$ in diameter. The ragi was prepared from rice flour with distinctive spices and contained the required organisms. Finally, the substrate is covered with a banana leaf and then allowed to ferment for approximately 24 to $48 \mathrm{~h}$ at ambient temperature $\left(25-30^{\circ} \mathrm{C}\right)^{[1,2,3,4,5]}$.

Once TK is prepared in packaging, the challenge to present TK in good condition increases as a distance from the markets increase, to fulfill the consumer demands. Since it is consumed directly as a dessert or a snack after the fermentation takes place, without further preparation. Generally, the fermentation state of TK was predicted based on fermentation time (2-3 days) for "ready to eat" food. However, it is not easy for consumers to determine the preferred fermented state quality. This is due to the fact that consumers have difficulty to determine when the fermented state of TK has reached their preferred state to be consumed. Thus, this condition became a barrier to show "best date" consumption for consumers.

The number of publications reported on package indicators for fermented products is still limited. However, a color indicator for kimchi packaging (fermented vegetable product) has been developed to monitor its quality during storage and distribution ${ }^{[6]}$. The film consist of polypropylene (PP) resin, $\mathrm{a} \mathrm{CO}_{2}$ absorbent using $\mathrm{Ca}(\mathrm{OH})_{2}$ and indicator, i.e. bromocresol purple or methyl red was attached to the lid. During fermentation of kimchi at 0,10 , and $20^{\circ} \mathrm{C}$, it's quality was evaluated for $\mathrm{pH}$ and titratable acidity (TA). Then, two parameters had been correlated with Hunter color values of the indicators. The results show the color values of the indicators correlated well with $\mathrm{pH}$ and TA values, regardless temperature of fermentation. Thus, the $\mathrm{pH}$ indicator (bromocresol purple or methyl red) was employed in monitoring quality of fermented product based on the $\mathrm{pH}$ change (the total acidity). However, other progress in relation to monitoring quality and safety of food, in term of novel packaging technology, such as smart packaging, have been reported elsewhere ${ }^{[7,9,10]}$.

Using similar principle, the $\mathrm{pH}$ indicators could also be employed for quality monitoring of fermented products, such as TK at their fermentation stage. Other approach could be proposed for this fermented product packaging is based 
on their water vapor (humidity). Since during fermentation process of TK, water vapor is produced and consumed along with the change of other parameters ( $\mathrm{pH}$, titratable acidity (TA) and ethanol). The purpose of this study is to use Nafion/ crystal violet (CV) film to construct on-package low-cost color indicator for determining the state of fermented TK. Since, the Nafion/CV film is highly sensitive to water vapor (relative humidity) ${ }^{[11,12,13]}$. Since the sulfonate groups of Nafion are highly acidic, so that the $\mathrm{CV}$ is in the acid environment. The water content in the film, will dictate the acidity of the proton bound to the sulfonate group. In a dry Nafion/CV film, the Nafion acidity is very strong, as a consequence, CV is in the diprotonated form, and make the film remains yellow. When water content increase in the film, the sulfonate groups become less acidic and protons loss from the diprotonated $\mathrm{CV}$, converting to the monoprotonated and non-protonated forms, which in turn, the color of the film changes to green ${ }^{[11]}$.

The aim of this work is to develop on-package color indicator based on Nafion/CV film for tape ketan packaging. Here, the color film changes from yellow to blue and pale blue, as a result of its interactions with water vapor produce during fermentation process, and were evaluated directly with color image analysis (CorelDraw). Furthermore, the change of color indicator was correlated with other parameters, such as $\mathrm{pH}$, titratable acidity (TA), ethanol content and sensory evaluation of TK samples during storage at ambient temperature.

\section{Materials and Methods}

\subsection{Chemicals}

A Crystal Violet (98\%) and Nafion solution (5 wt.\%) in low alcohols and 10\% water, were purchased from Aldrich (Milwaukee, WI, USA) and used as received. A stock standard solution of Crystal Violet (1000 ppm) was prepared in ethanol absolute (Aldrich, USA).

\subsection{Color Indicator Preparation}

Nafion/CV films were prepared as described previously ${ }^{[1]}$ with slight modification. Solutions of Crystal Violet (1000 ppm) in ethanol and Nafion were mixed together to make Nafion: Crystal Violet molar ratio at 1:1. Then the mixture was stirred (30 min) till homogenous. A $50 \mu \mathrm{L}$ solution drop was placed on the plastic disc to make the film ( $\sim 5 \mathrm{~mm}$ thickness), and then a $50 \mu \mathrm{L}$ drop of methanol was pipetted on to the film and allowed to dry for $30 \mathrm{~min}$ at $28 \pm 1{ }^{\circ} \mathrm{C}$. Afterward, the film is ready to be used as a color indicator. The design of the color indicator can be given in Figure 1(a).

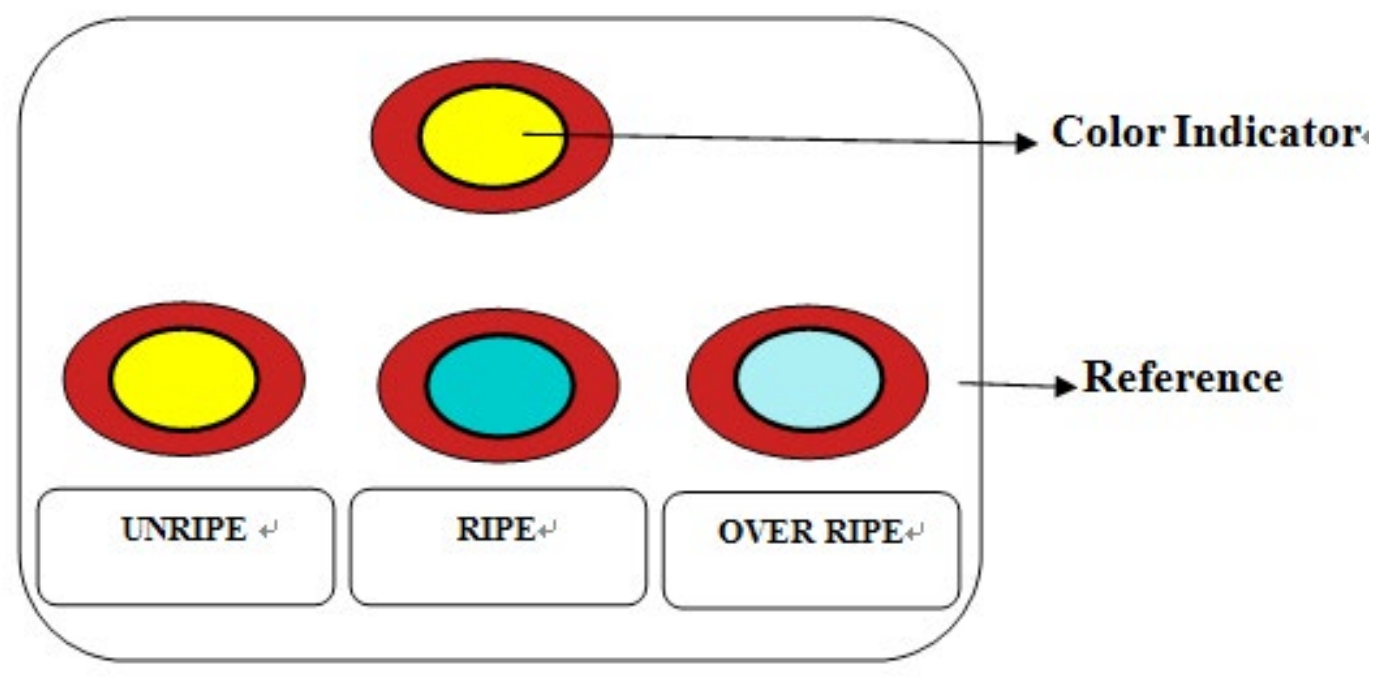

\subsection{TK Preparation}

Figure 1 (a). Design of color indicator for TK ripeness

The TK was prepared as described previously ${ }^{[14,15]}$. After each 100 -g portion of glutinous rice (White polished rice from Jember traditional market) was steamed and cooling, it was inoculated with the ragi tape from Jember, Indonesia. The inoculated steamed rice was then covered with a banana leaf and put in the PP tray with Ny/CPP lid. Finally, it allowed being fermented for approximately 24 to $48 \mathrm{~h}$ at ambient temperature $\left(28 \pm 1^{\circ} \mathrm{C}\right)$. Due to the fact that the temperature would affect the fermentation process of TK. Therefore, this ambient temperature was used for further experiments, in order to 
reflect the real condition that occurs in the store, supermarket, and home.

\subsection{Measurement and Analysis of the Color Indicator}

The measurement of the color indicator was described previously ${ }^{[16]}$ and their principles have been reported elsewhere ${ }^{[17,18,19]}$. The color indicator placed inside the lid of TK packaging as given in Figure 1(b). Using this design, the color change of the indicator can easily be detected by the naked eye during the fermentation stage. For quantitative measurement, a simple method was used, by means of a digital camera, rather than used the standard colorimetric method. In addition, this method shows its compatibility to be used with commercial devices, such as digital cameras and commercial software for image analysis. For color analysis, a digital camera (Canon MP 190, Japan) was used according to previously reported procedures using the graphics software CorelDraw ${ }^{[16]}$. This method is more suitable and simpler in comparison with visual inspection by the naked eye.
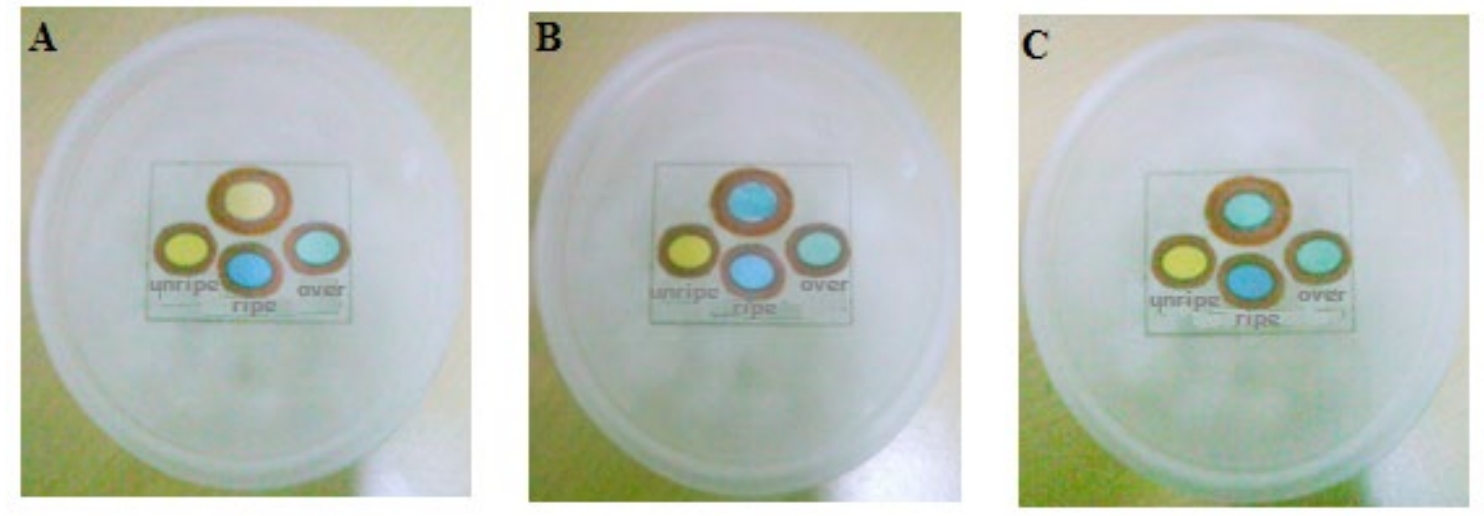

Figure 1 (b). The placement of color indicator inside the lit of package for TK ripeness stages, $A$ is unripe, $B$ is ripe and $C$ is overripe

\subsection{Measurement of Humidity, pH, TA, and Ethanol}

The determination of humidity inside TK packaging was measured with a commercial humidity meter (Hertz, UK KM8006). For proximate wet analyses, a 10 grams sample of TK was blended with distilled water $(20 \mathrm{ml})$ in a homogenizer for about 30 seconds. Then, the $\mathrm{pH}$ of the slurry was determined by a digital $\mathrm{pH}$ meter (Russel, Model RL150, USA). Titratable acidity of TK was determined by titrating the filtrates, a $10 \mathrm{~g}$ well-blended sample in $90 \mathrm{ml}$ distilled water with $0.1 \mathrm{~N} \mathrm{NaOH}$ to the endpoint using phenolphthalein as indicator. The alcohol content was determined using the distillation method, it was performed by blending $10 \mathrm{~g}$ sample with $90 \mathrm{ml}$ distilled water in the homogenizer as described above. Each sample was analyzed in triplicates, and the results were reported as mean values.

\subsection{Sensory Evaluation}

The sensory evaluation was used in this case, due to the fact that it shows the customer preferences toward TK ripeness stage to be more human and realistic, compared when the gas chromatography method used to detect aroma release during the fermentation stage, that can not be directly used to represent customer preferences. In order to describe TK ripeness stage by sensory evaluation, an additional test was performed ${ }^{[20]}$. For sensory evaluation, TK samples were investigated up to 7 days at ambient temperature. Every day, during a period of investigation, three of TK samples were taken from the package, tested and scored by a panel consisting of ten peoples (four males and six females with age 2030 years). The grading system was based on scores from 1 to 5 scales in categories of aroma, taste and color scores. Furthermore, the prevailing subjective status of the ripeness stage was evaluated from unripe to overripe. The samples were tested by individual taster independently, and the mean value of the scores was calculated. Finally, color change of the indicator and the evolution of other parameters ( $\mathrm{pH}$, acidity and ethanol) in the TK sample were investigated every day during the period of the investigation.

\subsection{Statistical Analysis}

The results of analyzed TK using the proposed on-package color indicator and its correlation with other parameters, such as RH, pH, titratable acidity (TA), and ethanol content as well as sensory evaluation are analyzed by using Pearson correlation coefficient $(\mathrm{R})^{[21]}$. 


\section{Results and Discussion}

\subsection{The Indicator Response Towards the Fermentation Stage}

All color indicators were placed inside the lid of packaging, in close proximate $(\sim 5 \mathrm{~cm})$ with TK samples to response to the relative humidity change during the fermentation process of TK, where the color indicator show a distinct color change from yellow to blue (Figure 1(b)). The other color indicator used as a blank was placed outside on the top of the lid, and expose to the ambient humidity during the period of investigation.

Since we used ragi tape as inoculum for the production of TK, the ragi tape that contains numerous yeasts, fungi and bacteria $^{[5,22,23,24]}$, are living aerobically or by fermentation. In the early stage of TK fermentation, enzymes are worked to produce free sugars ${ }^{[14,22]}$. This fact explains the hydrolysis of enzyme, that makes TK is sweet, were in this stage, the color indicator still yellow, meaning no vapor is released yet, as humidity inside packaging roughly the same. Humidity inside TK packaging has mainly resulted from the equilibrium between water contained in the TK and its headspace inside packaging since the headspace outside the packaging is not much affected due to the TK stored in tray with lit that has very limited or very low of water vapor transmission rate so that humidity outside packaging can be neglected. When alcohols are produced, by sugars metabolism without oxygen with the yeasts via fermentation process ${ }^{[25]}$, the color indicator starts to change to blue as water vapor also produces, and humidity inside packaging starts to increase drastically.

The fermentation process of TK is slightly different from the fermentation process of wine or beer, even though it used sugars to produce alcohol. In TK fermentation, it involves the process called solid substrate fermentation ${ }^{[23,24]}$. Each of the steamed glutinous rice is covered with a thin layer of viscous liquid, that makes diffusion and penetration of oxygen difficult. Thus, it makes a good environment setting for the fermentation of alcohol. The sugar molecules action with the liquid caused rheological changes of TK, which in turn, making it viscous or sticky. This is representing to the hydrogen bonding interaction between water molecules and the sugar molecules. The low content of alcohol in TK is due to not tight packing or the slightly open with high temperature inside packaging, that could result in the alcohol volatilization or vaporization and water vapor to the ambient environment inside packaging ${ }^{[26]}$, which in turn caused color indicator change to blue as humidity was higher.

After all the alcohols were produced as the products of fermentation is short-chain and lowering the evaporation, which in turn caused low water vapor as the color indicator change to pale blue, since the humidity was also lower. While, other color indicator used as blank placed outside on the top of the lid, was still yellow as no much change in the ambient humidity during the period of investigation. Here, the color image analysis using CorelDraw is presented as $\Delta$ mean green versus relative humidity (\%), as given in Figure 2 . The $\Delta$ mean green value is the difference between the average green value before and after exposure to relative humidity at certain stage of TK fermentation, and the $\Delta$ mean green used, as it fits with color change of the indicator towards the relative humidity inside TK packaging, compared to other color image analysis such as $\Delta$ mean blue, red and RGB. Therefore the $\Delta$ mean green was used for further measurements as color indicator response. 


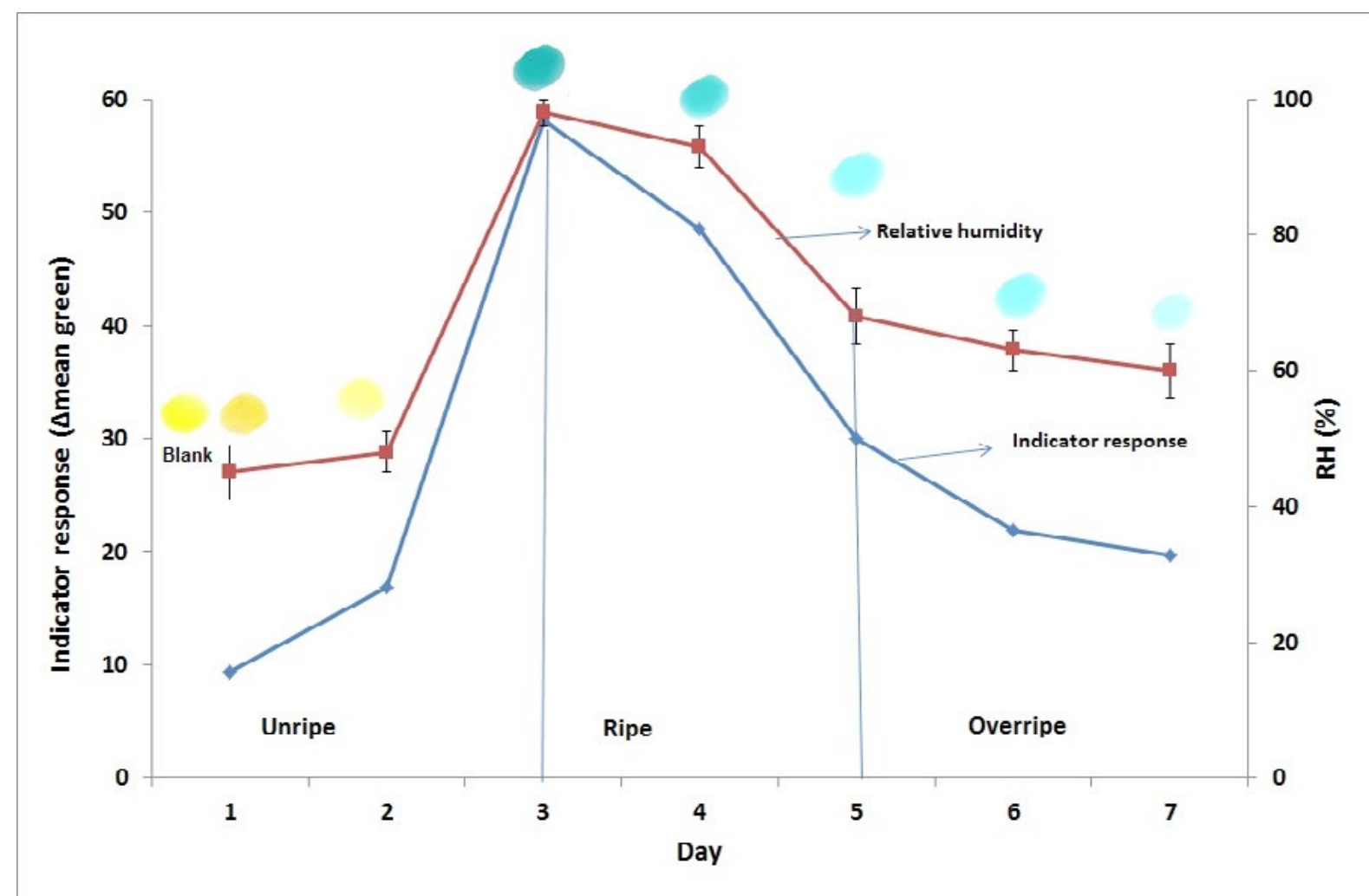

Figure 2. The color indicator response as $\Delta$ mean green vs. relative humidity (\%) during fermentation stage of TK within 7 days of investigation

\section{$3.2 \mathrm{pH}$, Acidity \& Ethanol}

Figure 3(a) shows the color indicator response vs. the $\mathrm{pH}$ values of the TK samples during the fermentation stage. The $\mathrm{pH}$ values of the TK samples were varied from $\mathrm{pH} 5.42$ at the unripe stage to $\mathrm{pH} 3.64$ at an over-ripe stage at seven days of the experiment at ambient temperature. It can be seen from Figure 3(a) that the indicator varies in the earlier or unripe stage, then follow a similar trend in the ripe stage until the overripe stage of the TK sample. However, even the indicator response presented as the $\Delta$ mean green in earlier stage or unripe is out of line with the $\mathrm{pH}$ decrease, however, the color indicator has a different color for each stage. It means that the decrease of $\mathrm{pH}$ during the TK fermentation process was followed by the color indicator change from yellow to blue and pale blue that reflects the fermentation stage, i.e. unripe, ripe and overripe respectively. 


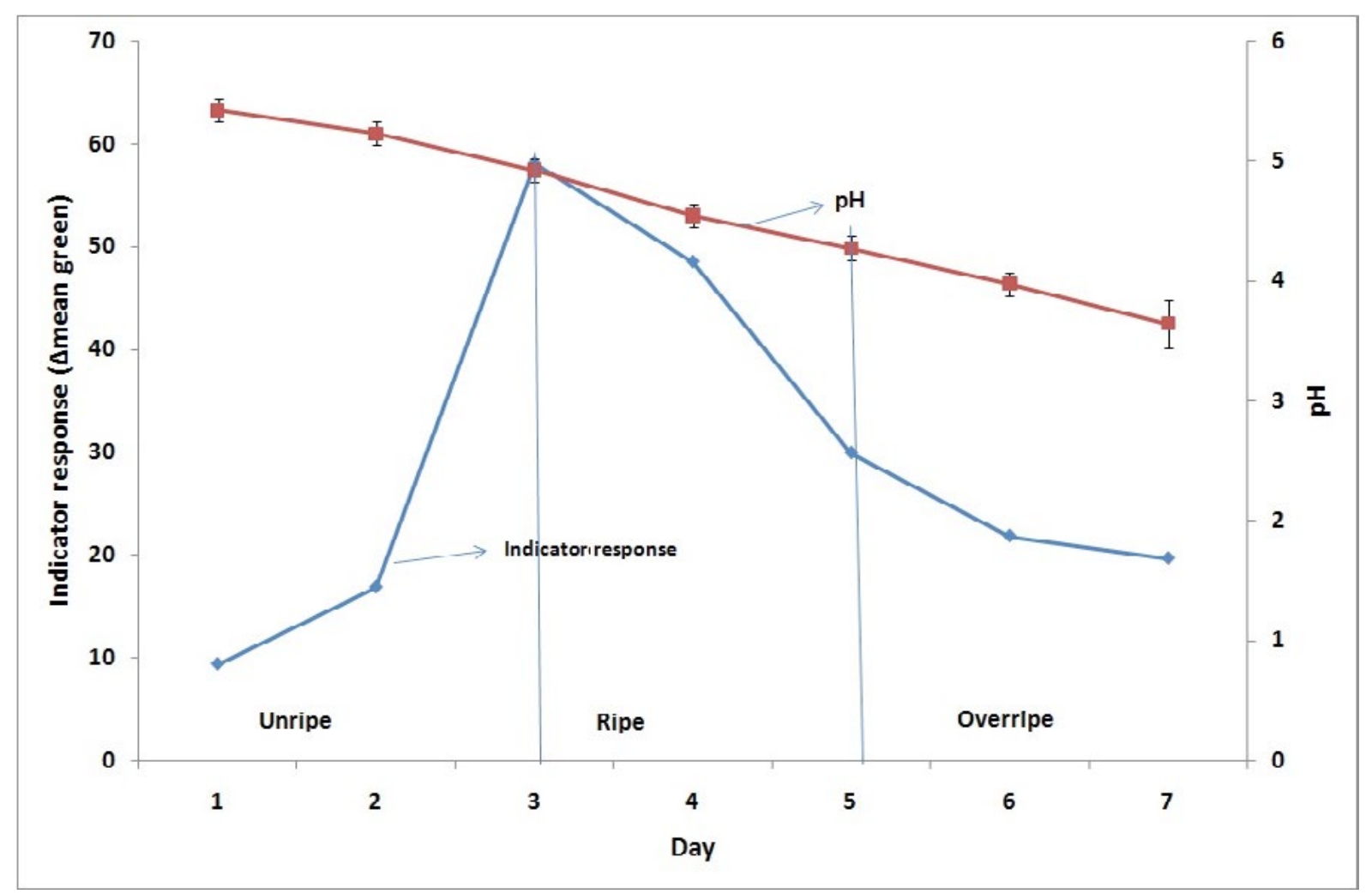

Figure 3 (a). The color indicator response vs. pH during fermentation stage of TK within 7 days of investigation

During the TK fermentation process ( 7 days in ambient temperature), the increase of titratable acidity ( $\% \mathrm{w} / \mathrm{v})$ from 0.08 to $0.76 \%$ in 7 days was given in Figure 3(b). It can be seen from Figure 3(b) that the indicator follows a similar trend until the TK ripe and vary from the acidity response when TK is ripe to overripe. However, even the indicator response is out of line with the acidity increase in overripe stages, but the color indicator also changes from yellow to blue then to pale blue when the acidity increase. It means that the increase in acidity during the TK fermentation process was indicated by the color change of indicator from yellow to blue and pale blue that represents unripe, ripe and overripe stages respectively. 


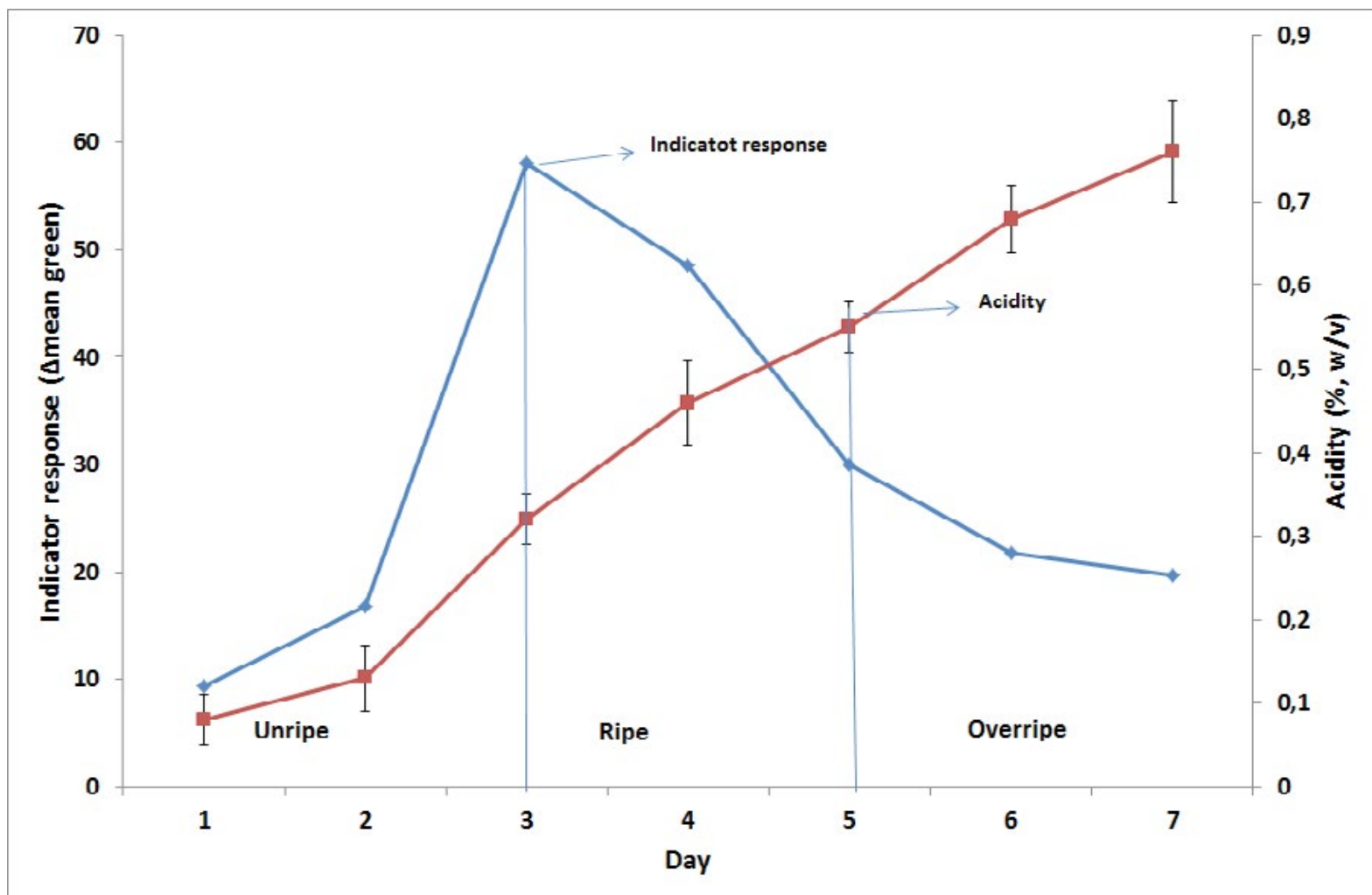

Figure 3 (b). The color indicator response vs. acidity (\%, w/v) during fermentation stage of TK within 7 days of investigation

The acidity increased during the TK fermentation process is similar results to the results of reported earlies ${ }^{[3,22]}$. The correlation between $\mathrm{pH}$ and acidity is believed to be associated with both lactic acid bacteria and yeasts contained in ragi $[3,24,27]$. Both were well known for the production of acids especially lactic acid, whereas previously some yeasts were reported to produce acid in the fermentation of alcohol to make a nice contribution to the flavor of products ${ }^{[24,28]}$. At this condition, high acidity and low $\mathrm{pH}$ would also eliminated coliforms, enteropathogen and spoilage organisms in this TK product $^{[3]}$.

Meanwhile, the alcohol content in TK increased day by day during fermentation and was found as high as $4.6 \%$ (v/ v) after 7 days (Figure 3(c)) as also reported in reported in literature ${ }^{[25]}$. Figure 3(c) shows that the indicator follow similar trend till the TK ripe and vary from the alcohol response, when TK in the ripe and overripe stages. However, even the indicator response is out of line with the alcohol increase in ripe to overripe stage, but the color indicator give different color for different stages. This means that the increase of alcohol content during TK fermentation process was followed by the color indicator change from yellow to blue and pale blue that represent from unripe, ripe and overripe state respectively. Thus, the color change of the indicator could be correlated with the decreasing of $\mathrm{pH}$ and increasing of acidity and ethanol, as its show by the color indicator change from yellow (unripe) to blue (ripe) and pale blue (unripe) respectively. 


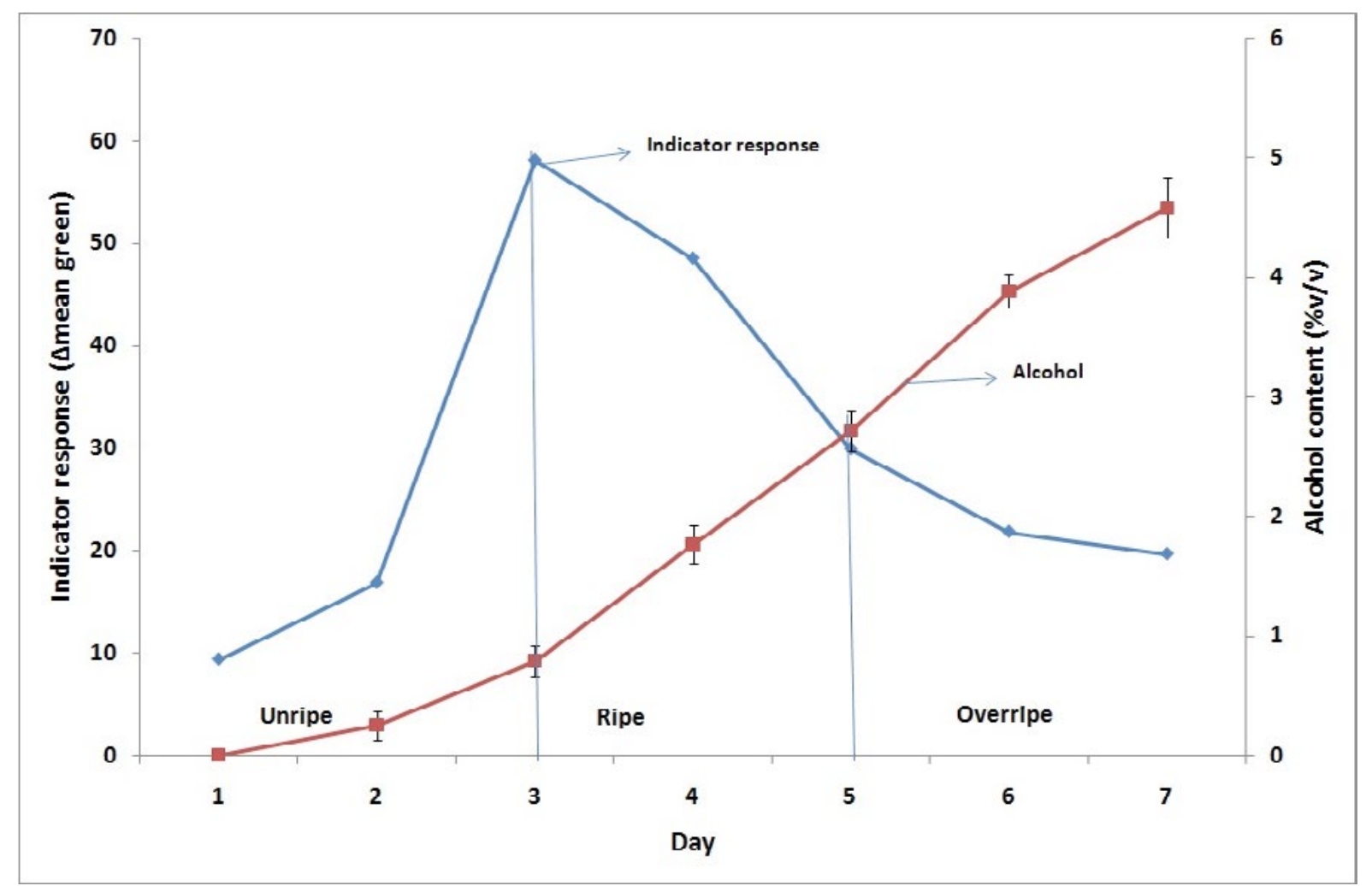

Figure 3 (c). The color indicator response vs. alcohol content (\%v/v) during fermentation stage of TK within 7 days of investigation

In the fermentation process, alcohol produced plays an important role in helping to extract flavor compounds from the substrates. Both lactic acid bacteria and yeasts also produce other volatile compounds, e.g. malty flavored 2-methyl-propane ${ }^{[3,24]}$. Hence, correlation assessment between RH, pH, acidity, and alcohol content provides the potential application of the porposed on-package color indicator label in assessing TK ripeness. Pearson correlation coefficient (R) showed a high and positive correlation between $\Delta$ mean green and $\mathrm{RH}$, as well as $\mathrm{pH}$ after TK ripeness has been reached. While a high and negative correlation between $\Delta$ mean green and acidity as well as ethanol content has been achieved after TK ripeness. According to the multiple regression analysis with fitting line and $\mathrm{r}$ (presented in Table 1) were calculated for $\mathrm{RH}, \mathrm{pH}$, acidity, and TMC. Therefore, RH, pH, acidity, and ethanol content parameters showed the high potential application of the on-package color indicator to asses the ripeness of TK.

Table 1. Pearson correlation coefficient of $\Delta$ mean green of color indicator with TK qualities' parameters

\begin{tabular}{lc}
\hline Parameters & Pearson's r \\
\hline RH & $0.975^{*}$ \\
Total acidity (after ripe) & $0.970^{*}$ \\
pH (after ripe) & $0.961^{*}$ \\
Alcohol content (after ripe) & $0.966^{*}$ \\
\hline "represents that the values are significantly different (p $\leq 0.05)$.
\end{tabular}

\subsection{Sensory Analysis}

The aroma, taste, and color of TK samples were also evaluated by sensory evaluation. The evaluations were conducted along with the color indicator response, and the results were also recorded. The sensory evaluation, therefore, confirmed the results of the color indicator response. The evaluation was performed in the laboratory conditions without any special treatments, considering the application of the color indicator at the shopping center, restaurant, stores, and others. Figure 4 shows the output score of the aroma (a), taste (b) and color (c) of TK samples evaluations are also presented in Table 2. Table 2 lists the results of the sensory evaluation in ambient temperature vs. indicator response. From Figure 4, it can be noticed that the color indicator change has a similar response to the sensory score (aroma, taste and color score), where the point of the high acceptance of sensory score was similar to the optimum response of the color indicator response. While, correlation assessment between sensory evaluation (aroma, taste and color) provides the potential application of 
the porposed color indicator label in assessing TK ripeness. Pearson correlation coefficient (R) showed a high and positive correlation between $\Delta$ mean green and sensory evaluation. Based on the multiple regression analysis with fitting line and $r$ (presented in Table 3) were calculated for sensory evaluation (aroma, taste and color ). Therefore, sensory evaluation parameters presented the high potential application of the color indicator label to evaluate the ripeness of TK.

Table 2. Sensory evaluation results vs. indicator response of TK during fermentation process

\begin{tabular}{|c|c|c|c|c|}
\hline Day & Aroma & Taste & Color & $\Delta$ Mean Green \\
\hline 1 & $1.3 \pm 0,5$ & $1.2 \pm 0.4$ & $1.5 \pm 0,5$ & $9.36 \pm 0.1$ \\
\hline 2 & $2.2 \pm 0.6$ & $2.4 \pm 0.5$ & $2.6 \pm 0.5$ & $16.86 \pm 1$ \\
\hline 3 & $4.8 \pm 0.6$ & $4.9 \pm 0.3$ & $5.0 \pm 0.5$ & $58.12 \pm 0.1$ \\
\hline 4 & $4.5 \pm 0.5$ & $4.5 \pm 0.5$ & $4.6 \pm 0.5$ & $48.53 \pm 1$ \\
\hline 5 & $2.7 \pm 0.5$ & $2.6 \pm 0,5$ & $2.7 \pm 0.5$ & $29.95 \pm 1$ \\
\hline 6 & $1.8 \pm 0.5$ & $1.6 \pm 0.5$ & $1.6 \pm 0.5$ & $21.84 \pm 1$ \\
\hline 7 & \pm 0.3 & & 1.0 & $19.66 \pm 0.6$ \\
\hline
\end{tabular}

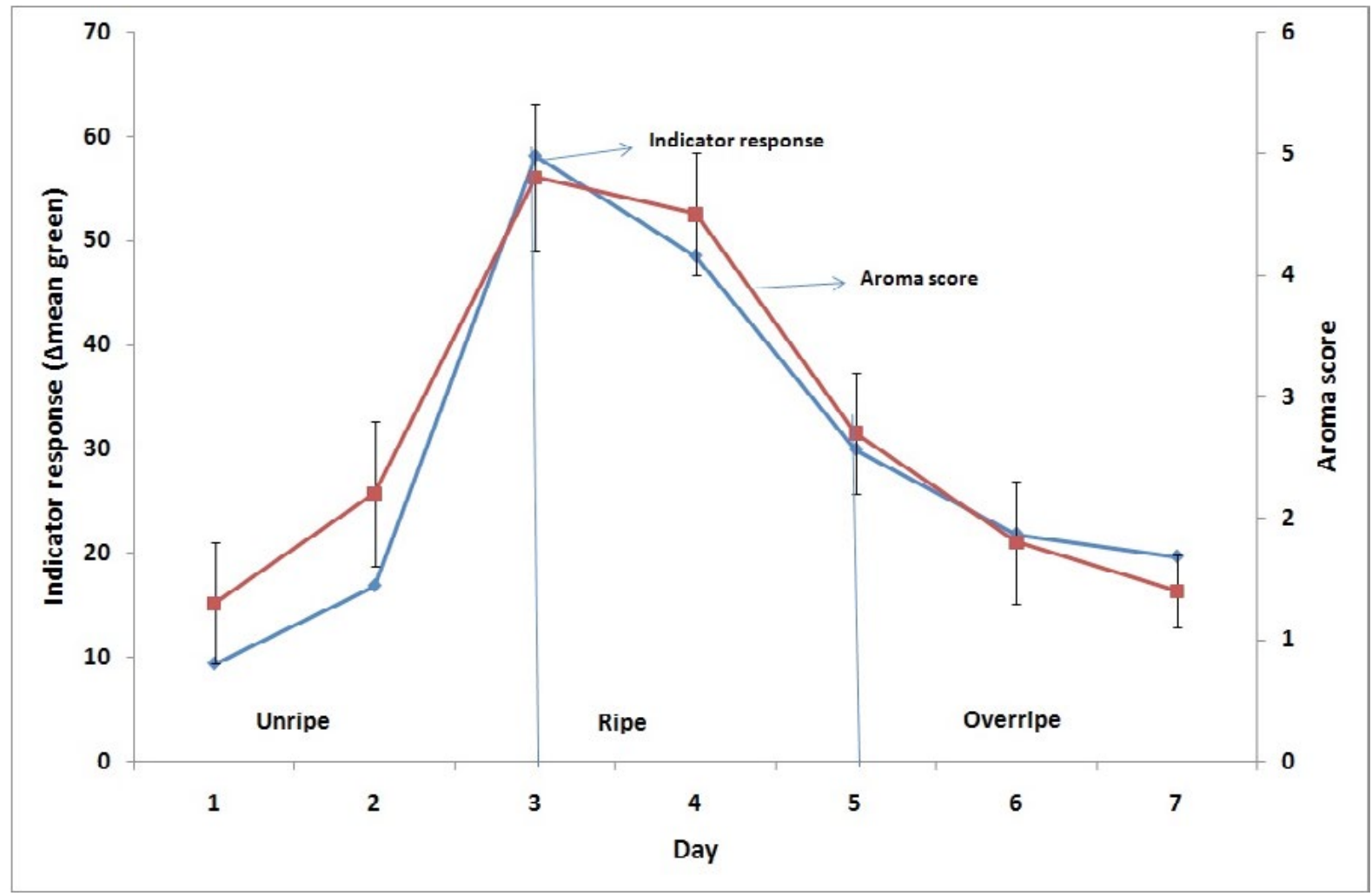

(a) 


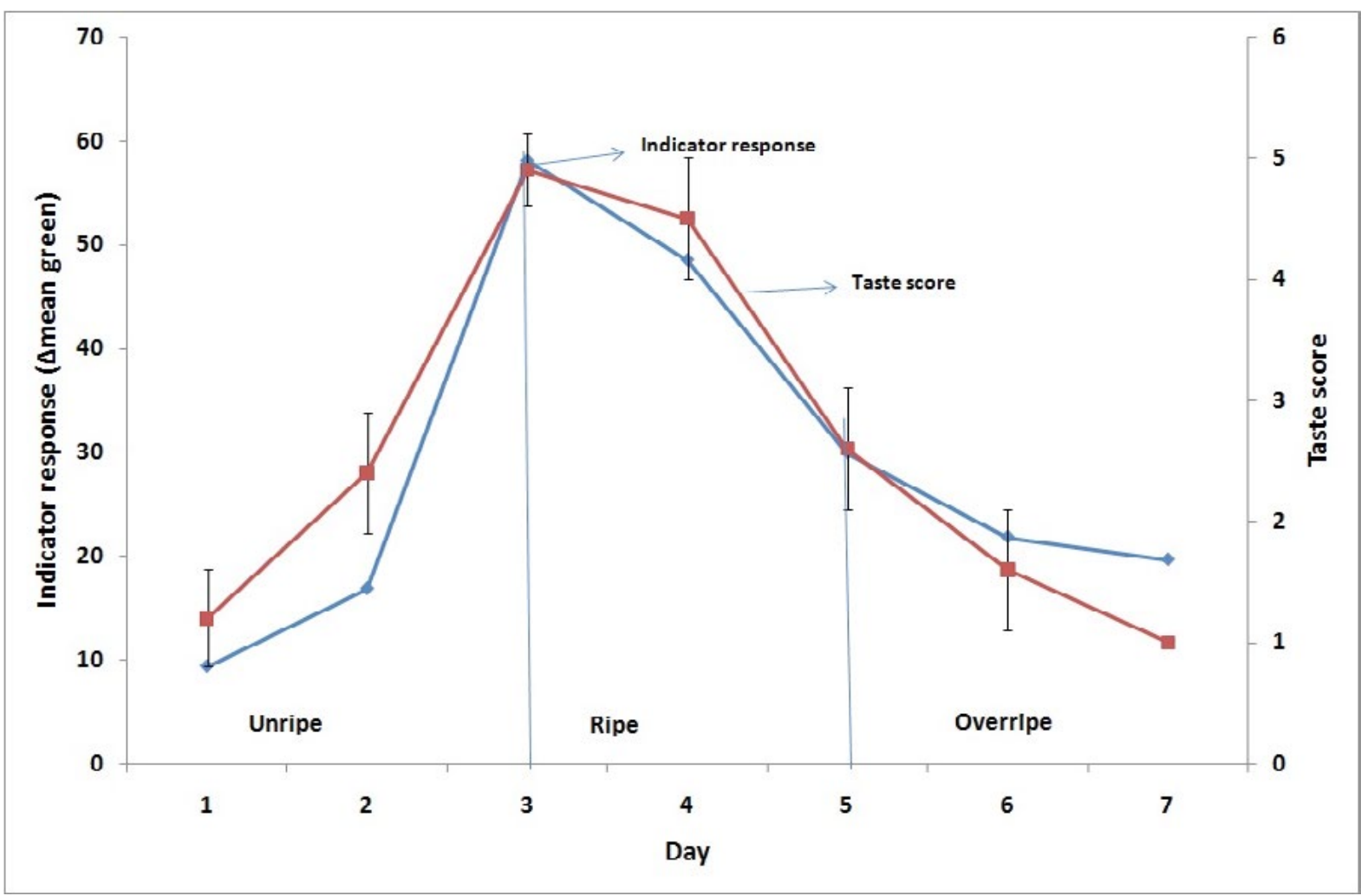

(b)

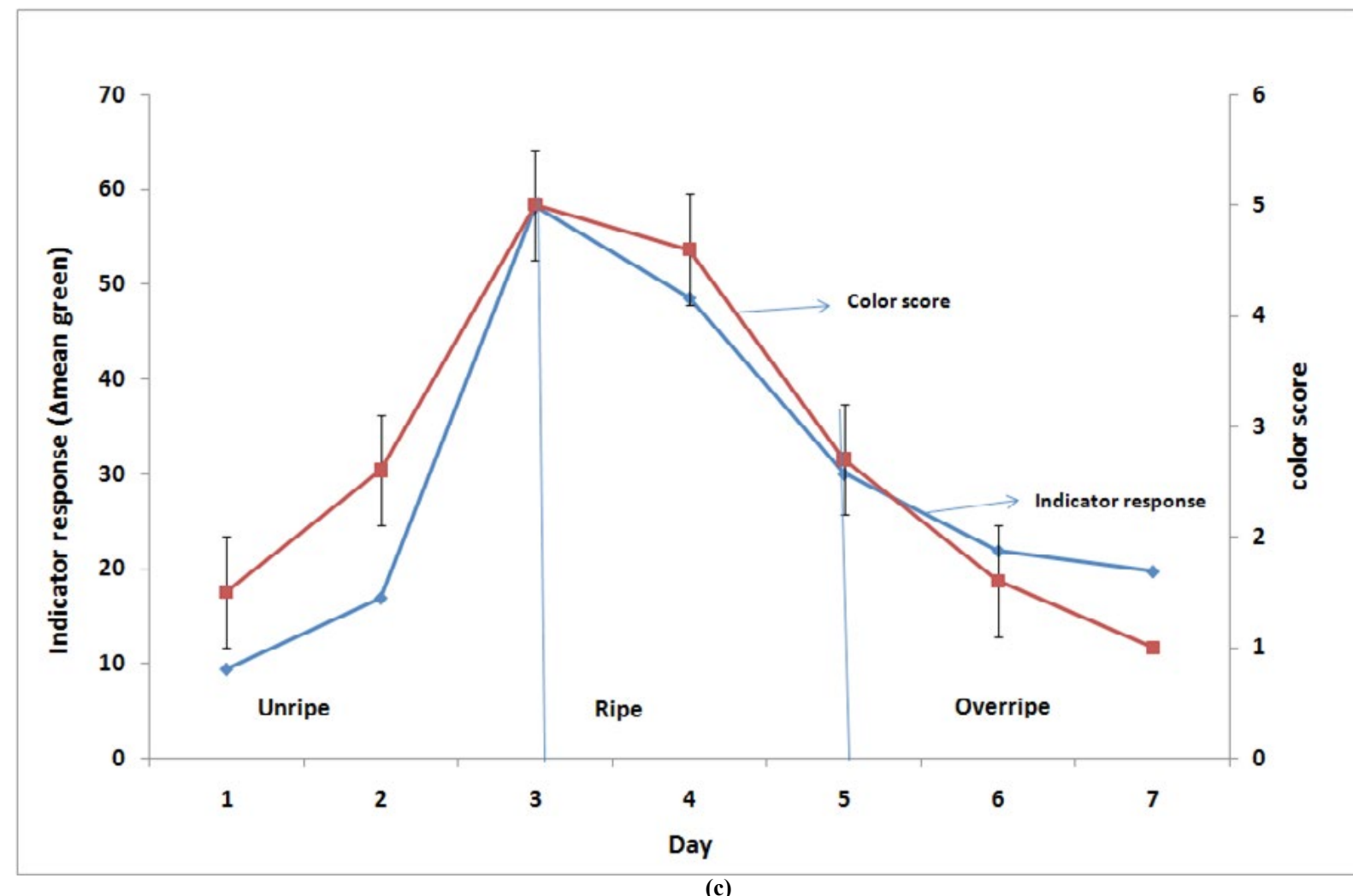

Figure 4. The color indicator response vs. aroma score (a); taste score (b); color score (c) during fermentation stage of TK within 7 days of investigation 
Table 3. Pearson correlation coefficient of $\Delta$ mean green of color indicator with TK Sensory's evaluation results

\begin{tabular}{lc}
\hline Parameters & Pearson's r \\
\hline Aroma & $0.960^{*}$ \\
Taste & $0.941^{*}$ \\
Color & $0.921^{*}$ \\
\hline represents that the values are significantly different $(\mathrm{p} \leq 0.05)$.
\end{tabular}

Here, the color indicator response fits well with the sensory response, compared to $\mathrm{pH}$, acidity and ethanol content, since the flavors in TK product yield unique fermented products in term of aroma, taste, and color ${ }^{[2,5]}$ and these parameters scored by panels, which were much different from the unfermented (unripe) and over fermented (overripe) products of TK. Therefore, the color indicator can aid customers to select easily, which is fermented stage of TK product, that they prefer, for instance, unripe if the TK product would like to be consumed within 2-3 days later, ripe for immediate consumed within one day or next day, and overripe for those, who like higher alcohol content of TK product and warning of nearly expired product for the sellers. Thus, the on-package color indicator changes represented the ripeness stage of TK products, which could be used as an alternative for an effective non-destructive smart label for online monitoring of TK ripeness during the fermentation process.

\section{Conclusion}

A simple Nafion/CV film was employed as an on-package color indicator and the correlation between the indicator and TK fermented stage was studied. The results of the study show that the color indicator could be employed for evaluation of TK fermented stage since the correlation between the indicator color change towards the fermentation stage of TK is in similar trend with sensory evaluation score, and the fermentation stage of TK samples could be monitored clearly via the color change of the indicator from yellow color when unripe, blue color when ripe, and finally pale blue color when overripe. The color indicator responses toward water vapor/humidity change in the package headspace during the fermentation process of TK packaging, which can be correlated with other parameters, such as $\mathrm{pH}$, TA, and ethanol. Thus, the color indicators could be used as an alternative for the effective non-destructive smart label for on-package ripeness monitoring of fermented TK products. For the consumers, by viewing the color of the indicator, it could help to choose TK at their preferred state of fermentation. Further studies are required on the color indicators for TK using different sensing schemes, such as $\mathrm{pH}$ and ethanol. This smart label might be of potential value in assessing failed packages of fresh foods, e.g. minimally processed fruits and vegetables, where their freshness related to the water vapor released inside the packaging. Since, the color indicator may serve as "smart labeling" devices coupled with the "used-by-date" labeling, when attached to each packaged product, or could be used in helping to optimize and manage the distribution control and the stock rotation system, which in turn, could reduce the food waste.

\section{Acknowledgment}

The authors gratefully thank the DRPM, Ministry of Education and Culture, the Republic of Indonesia for supporting this work via the Basic Research Grant 2019 (Hibah Riset Dasar Tahun 2019).

\section{References}

[1] K.E. Aidoo, M.J. Rob Nout, P.K. Sarkar. Occurrence and function of yeasts in Asian indigenous fermented foods. FEMS Yeast Res. 2006; 6 (1): 30-39. https://doi.org/10.1111/j.1567-1364.2005.00015.x.

[2] A. Bakar, M. Hashim, A. Hamid. MiniReview Popular fermented foods and beverages in Southeast Asia. Int. Food Res. J. 2011; 18 (1): 475-484.

[3] Y.W. Chiang, M. Ismail. Microbial Diversity and Proximate Composition of Tapai, A Sabah's Fermented Beverage, Malays. J. Microbiol. 2006; 2: 1-6.

[4] L.R. Beuchat. Indigenous Fermented Foods, Biotechnol. Set. 200, 505-559. https://doi.org/10.1002/9783527620999. $\operatorname{ch} 13 \mathrm{j}$.

[5] S. Siebenhandl, L.N. Lestario, D. Trimmel, et al. Studies on tape ketan--an Indonesian fermented rice food. Int. J. Food Sci. Nutr. 2001; 52 (1): 347-57. http://www.ncbi.nlm.nih.gov/pubmed/11474900 (accessed June 13, 2016).

[6] S.-I. Hong, W.-S. Park. Development of Color indicators for kimchi packaging. J. Food Sci. 1999; 64 (1): 255-257. 
https://doi.org/10.1111/j.1365-2621.1999.tb15877.x.

[7] K.B. Biji, C.N. Ravishankar, C.O. Mohan, et al. Smart packaging systems for food applications: a review. J. Food Sci. Technol. 2015; 52 (13): 6125-6135. https://doi.org/10.1007/s13197-015-1766-7.

[8] D. Schaefer, W.M. Cheung. Smart Packaging: Opportunities and Challenges. Procedia CIRP. 2018; 72 (3): 10221027. https://doi.org/10.1016/j.procir.2018.03.240.

[9] P. Müller, M. Schmid. Intelligent Packaging in the Food Sector: A Brief Overview. Foods. 2019; 8 (1): 16. https://doi. org/10.3390/foods 8010016 .

[10] M. Rai, A.P. Ingle, I. Gupta, et al. Smart nanopackaging for the enhancement of food shelf life. Environ. Chem. Lett. 2019; 17 (1): 277-290. https://doi.org/10.1007/s10311-018-0794-8.

[11] I.M.R. Jr, R. Narayanaswamy. Evaluation of Nafion-Crystal Violet films for the construction of an optical relative humidity sensor, Analyst. 1999; 1: 1623-1627. https://doi.org/10.1039/a905264j.

[12] T.E. Brook, M.N. Taib, R. Narayanaswamy. Extending the range of a fibre-optic relative-humidity sensor. Sensors Actuators B Chem. 1997; 39 (10): 272-276. https://doi.org/10.1016/S0925-4005(97)80217-1.

[13] Y. Sadaoka, Y. Sakai, Y. Murata, Q. Zhou, et al, Optical properties of cresyl violet-polymer composites for quantification of humidity and ammonia gas in ambient air. J. Mater. Chem. 1993; 3 (1): 247-251. https://doi. org/10.1039/JM9930300247.

[14] D. Dwidjoseputro, F.T. Wolf. Microbiological studies of Indonesian fermented food stuffs. Mycopathol. Mycol. Appl. 1970; 41 (1): 211-222.

[15] K.O.S. Djien, N. A-. Tape Fermentation. Appl. Microbiol. 1972; 23 (1): 976-978.

[16] B. Kuswandi, C. Maryska, Jayus, et al. Real time on-package freshness indicator for guavas packaging. J. Food Meas. Charact. 2013; 7 (1): 29-39. https://doi.org/10.1007/s11694-013-9136-5.

[17] F.J. Francis. Colorimetric properties of foods, in: M.A. Rao, S.S.H. Rizvi (Eds.), Eng. Prop. Foods, 2nd ed. Marcel Dekker, Inc., New York, 1994; pp. 495-523.

[18] S. Gunasekaran. Computer vision technology for food quality assurance. Trends Food Sci. Technol. 1996; 7 (2): $245-$ 256. https://doi.org/10.1016/0924-2244(96)10028-5.

[19] K.L. Yam, S.E. Papadakis. A simple digital imaging method for measuring and analyzing color of food surfaces. $J$. Food Eng. 2004; 61 (1): 137-139.

[20] A. Abdullah. Prinsip Penilaian Sensori, 1st ed. Unri Press, Pekan Baru, 2005.

[21] F. Ebrahimi Tirtashi, M. Moradi, H. Tajik, et al. Cellulose/chitosan pH-responsive indicator incorporated with carrot anthocyanins for intelligent food packaging. Int. J. Biol. Macromol. 2019; 136 (6): 920-926. https://doi.org/10.1016/ j.ijbiomac.2019.06.148.

[22] T.C. Cronk, K.H. Steinkraus, L.R. Hackler, et al. Indonesian tapé ketan fermentation. Appl. Environ. Microbiol. 1977; 33 (1): 1067-1073.

[23] M. Z, Y. Quee-Lan. Industrialization of indigenous fermented foods, in: K.H. Steinkraus (Ed.). Marcel Dekker, Inc., New York. 2004; pp. 247-270.

[24] M. Ardhana, G.H. Fleet. The microbial ecology of tape ketan fermentation. Int. J. Food Microbiol. 1989; 9 (1): 157165. https://doi.org/10.1016/0168-1605(89)90086-X.

[25] T.C. Cronk, L.R. Mattick, K.H. Steinkraus, et al. Production of higher alcohols during indonesian tapé ketan fermentation. Appl. Environ. Microbiol. 1979; 37 (1): 892-6.

[26] A.S. Azmi, G.C. Ngoh, M. Maizirwan, et al. Ragi tapai and Saccharomyces cerevisiae as potential coculture in viscous fermentation medium for ethanol production. African J. Biotechnol. 2010; 9 (1): 7122-7127. https://doi. org/10.5897/AJB10.933.

[27] Z.-H. Lu, H.-H. Peng, W. Cao, et al. Isolation, characterization and identification of lactic acid bacteria and yeasts from sour Mifen, a traditional fermented rice noodle from China. J. Appl. Microbiol. 2008 ; 105 (1): 893-903. https:// doi.org/10.1111/j.1365-2672.2008.03814.x.

[28] G.H. Fleet. Yeast interactions and wine flavour. Int. J. Food Microbiol. 2003; 86 (3): 11-22. https://doi.org/10.1016/ S0168-1605(03)00245-9. 